\title{
Frequency components of systolic blood pressure variability reflect vasomotor and cardiac sympathetic functions in conscious rats
}

\author{
Takahiko Yoshimoto • Kunihiro Eguchi · Hiroki Sakurai • Yusuke Ohmichi • \\ Tatsuyuki Hashimoto • Mika Ohmichi · Atsuko Morimoto • Yoshiko Yamaguchi • \\ Takahiro Ushida $\cdot$ Satoshi Iwase $\cdot$ Junichi Sugenoya $\cdot$ Takao Kumazawa
}

Received: 19 March 2011 / Accepted: 6 June 2011/Published online: 29 June 2011

(c) The Author(s) 2011. This article is published with open access at Springerlink.com

\begin{abstract}
In this study, after confirming the suppression of autonomic nervous function by isoflurane anesthesia using autonomic antagonists, we pharmacologically investigated the involvement of vasomotor and cardiac sympathetic functions in systolic blood pressure variability (SBPV) frequency components in conscious rats at rest and during exposure to low-ambient temperature (LT-exposure, $9^{\circ} \mathrm{C}$ for $90 \mathrm{~min}$ ). Under unanesthesia, phentolamine administration $(\alpha$-adrenoceptor antagonist, $10 \mathrm{mg} / \mathrm{kg}$ ) decreased the midfrequency component (MF $0.33-0.73 \mathrm{~Hz}$ ) and inversely increased the high-frequency component (HF 1.3-2.5 Hz). The increased HF was suppressed by subsequent treatment
\end{abstract}

This study was conducted under Prof. Takao Kumazawa's supervision, but unfortunately he suddenly passed away on 26 July 2010 before completing this final manuscript. We dedicate this paper respectfully and affectionately to the memory of our esteemed teacher, Dr. Kumazawa.

T. Yoshimoto $\cdot$ K. Eguchi $\cdot$ H. Sakurai · Y. Ohmichi ·

T. Hashimoto $\cdot$ M. Ohmichi - A. Morimoto ·

Y. Yamaguchi · T. Kumazawa

Department of Algesiology, Aichi Medical University,

Nagakute, Aichi 480-1195, Japan

Present Address:

T. Yoshimoto $(\bowtie) \cdot S$. Iwase $\cdot$ J. Sugenoya

The Second Department of Physiology,

Aichi Medical University, Nagakute,

Aichi 480-1195, Japan

e-mail: tyos@aichi-med-u.ac.jp

K. Eguchi

Department of Physiology, Aichi Gakuin University,

Kusumoto, Nagoya 464-0037, Japan

T. Ushida

Department of Multidisciplinary Pain Center,

Aichi Medical University, Nagakute, Aichi 480-1195, Japan with atenolol ( $\beta$-adrenoceptor antagonist, $10 \mathrm{mg} / \mathrm{kg}$ ), but not with atropine (muscarinic receptor antagonist, $10 \mathrm{mg} / \mathrm{kg}$ ). Moreover, phentolamine administration after atenolol decreased MF, but did not increase HF. LT-exposure increased MF and HF; however, phentolamine pretreatment suppressed the increased MF during LT-exposure, and atenolol pretreatment dose-dependently decreased the increased HF. These results suggest that MF and HF of SBPV may reflect $\alpha$-adrenoceptor-mediated vasomotor function and $\beta$-adrenoceptor-mediated cardiac sympathetic function, respectively, in the conscious state.

Keywords Systolic blood pressure variability . Frequency analysis - Sympathetic nervous system . Conscious rat $\cdot$ Autonomic antagonist

\section{Introduction}

Blood pressure (BP) and heart rate (HR) continuously fluctuate over time under the influence of control mechanisms such as autonomic nerve activity, humoral factors and respiration for maintaining cardiovascular homeostasis. Among the methods used for analyzing these fluctuations, frequency domain analysis of cardiovascular variability has been used most often as an index of autonomic circulatory control [1]. In particular, heart rate variability (HRV) has become an increasingly popular index for the assessment of autonomic nervous system (ANS) characteristics such as vagal function or cardiac sympatho-vagal balance [2, 3]. However, when evaluating cardiac sympathetic regulation, HRV should be used with discretion since HRV merely reflects the relative value of sympatho-vagal balance; furthermore, the sympathetic and vagal functions are not always reciprocal under all conditions [4]. Systematic and 
discriminative assessment of vasomotor and cardiac sympathetic functions may be key to understanding complicated pathological conditions because activity of the sympathetic nervous system is regionally specific (heart, vessels, etc.). However, no reliable and accessible tool exists for simultaneous evaluation of vasomotor and cardiac sympathetic nervous function as separate measures.

Information regarding ANS provided by systolic arterial blood pressure variability (SBPV) is different from that provided by HRV. SBPV is commonly analyzed on the basis of low-, mid- and high-frequency components (LF, MF and HF, respectively) of the power spectrum. It has been reported that LF may be mediated by sympathetic and/or humoral factors including the renin-angiotensin system [5], and MF may show strong coherence with synchronous oscillations of post-ganglionic sympathetic nerve activity [6-8] and be decreased by $\alpha$-adrenoceptor blocker [9-11]. It has been suggested that the genesis of MF may involve in the sympathetic vasomotor component of the arterial baroreceptor reflex; however, it is not known exactly if MF is solely dependent on the $\alpha$-adrenoceptormediated sympathetic nervous system. Experiments under various physical conditions including external stimuli are thus necessary to make MF more applicable. The mechanism underlying HF has not been adequately explained despite several studies indicating that it is a mechanical consequence of respiration [12,13] and other studies suggesting an association between HF and ANS, particularly the $\beta$-adrenergic system [14-16]. To make progress in the availability of SBPV frequency analysis for the evaluation of sympathetic cardiovascular function, the association between the sympathetic nervous system and SBPV must be determined.

Most studies of the involvement of sympathetic cardiovascular function in SBPV (particularly in respiratory HF) are conducted under anesthesia [14-16]; therefore, little is known concerning the natural responses to stimuli under unanesthesia when ANS is not suppressed. It is meaningful to examine the ANS response in a conscious state in order to evaluate complicated pathological conditions (e.g., chronic pain) in human [17] and animal models [18-20].

We hypothesized that MF and HF in SBPV in the conscious state may be involved in the $\alpha$ - and $\beta$-adrenergic system, respectively. In the present study, to identify SBPV characteristics, we focused on changes in ANS in a conscious state and explored the link between ANS function and SBPV by means of sequential administration of autonomic antagonists and sympathetic activation stimulus. We first identified differences in autonomic nervous function between anesthetized and unanesthetized states using autonomic antagonists. Then, to elucidate the involvement of vasomotor and cardiac sympathetic functions in SBPV frequency components in conscious rats, we investigated responses to combined administration of autonomic antagonists at rest and in a systemic sympathetic activated state by exposure to low ambient temperature (LT-exposure). A strong relationship between SBPV and $\alpha$ - and $\beta$-adrenoceptor-mediated sympathetic function was revealed in conscious rats. Exploration in the frequency domain of cardiovascular regulation in animals could enhance our understanding of the pathology of autonomic dysfunction in humans. Our results suggest that SBPV may be used as a tool for detailed assessment of vasomotor or cardiac sympathetic functions.

\section{Materials and methods}

All experiments in this study were carried out according to a protocol approved by the Animal Care Committee at the Aichi Medical University and to the Fundamental Guidelines for Proper Conduct of Animal Experiments and Related Activities in Academic Research Institutions in Japan.

\section{Materials}

The experiments were performed using male SpragueDawley rats (300-400 g, SLC Inc., Japan). The rats were housed under controlled ambient conditions $\left(23 \pm 0.5^{\circ} \mathrm{C}\right.$, $50 \pm 10 \%$ ) with a $12-12 \mathrm{~h}$ light/dark cycle and provided food and water ad libitum. To minimize effects attributable to circadian alterations, all experiments in this study were conducted between 10 a.m. and 5 p.m. Recordings were performed in a silent room under controlled ambient conditions, as noted above.

\section{Autonomic blockers}

Phentolamine mesilate $(10 \mathrm{mg} / \mathrm{kg})$, a non-selective $\alpha$ adrenoceptor antagonist, was obtained from Novartis Pharma (Tokyo, Japan). Atenolol (1 and $10 \mathrm{mg} / \mathrm{kg}$ ), a selective $\beta 1$-adrenoceptor antagonist, and atropine methyl bromide $(10 \mathrm{mg} / \mathrm{kg})$, a non-selective muscarinic receptor antagonist, were purchased from Sigma (St. Louis, MO). Each drug was dissolved in saline and injected intraperitoneally (i.p.). To examine the effects of drugs on peripheral ANS, we chose these drugs that do not cross the bloodbrain barrier. The concentration of each drug $(10 \mathrm{mg} / \mathrm{kg})$ was determined in a pilot study: we ensured that each drug provided a sufficient effect over $60 \mathrm{~min}$ and gradual recovery $2-3 \mathrm{~h}$ after injection.

During this research, we recorded BP continuously for 3 days just after our manipulation (administration of drug or LT-exposure) and examined the effect of the 
manipulation on the sleep-wake cycle. From the next day after the manipulation, the BP fluctuation pattern returned to normal levels regardless of treatment type. Therefore, we presume that pharmacological manipulation in this study might not have affected the conscious state on the following day of the experiment.

Data acquisition and analysis of cardiovascular parameters

\section{Telemetric monitoring of arterial blood pressure and heart rate}

A telemetry system (Primtech Ltd., Japan) was used to continuously record arterial blood pressure (BP) in conscious rats. A radio transmitter equipped with a BP transducer (Physiotel TA11PA-C40, Data Science International, USA) was implanted as follows: (1) under pentobarbital anesthesia (50 mg/kg, i.p.), a catheter connected to the BP transducer was introduced into the abdominal aorta just below the renal artery, (2) the body of the transducer was fixed in the abdominal cavity, and (3) the incision was closed with sutures after antibiotics were sprayed into the abdominal cavity. Continuous recording of BP was initiated at least 1 week after operation in unrestrained and conscious rats.

Electrical signals transformed from BP of rats in each cage were detected by the receiver of the telemetry system, amplified using an electrical circuit in the receiver, and led to a 12-bit A/D converter (PCI-6040E, National Instruments, USA) that digitized the analog signals at a rate of $200 \mathrm{~Hz}$. The converter was controlled by a personal computer. The sampling procedure was programmed using LabVIEW 7.1 (National Instruments). The executable program was developed in our laboratory.

A maximum value corresponding to systolic arterial blood pressure (SBP) and its generation time, which was also utilized to calculate instantaneous heart rate (HR), for each pulse wave of BP were detected, and time series of SBP $\left(\mathrm{TS}_{\mathrm{SBP}}\right)$ and $\mathrm{HR}\left(\mathrm{TS}_{\mathrm{HR}}\right)$ were constructed. Subsequently, to create a power spectrum with a frequency range up to about $10 \mathrm{~Hz}$ by using a continuous wavelet transform, $\mathrm{TS}_{\mathrm{SBP}}$ with irregular intervals was reconstructed with a regular interval of $18.2 \mathrm{~Hz}$, so-called resampling.

For this procedure, we used the "Smooth Spline" function in the software $\mathrm{R}$ (http://www.r-project.org/), which is a freeware program used for statistics and signal processing.

\section{Frequency analysis of blood pressure fluctuations}

With resolutions of time and frequency of about $55 \mathrm{~ms}$ and $0.018 \mathrm{~Hz}$, respectively, the power of each frequency at each time point in the variability of $\mathrm{TS}_{\mathrm{SBP}}$ was calculated using a continuous Gabor wavelet transform of $\mathrm{TS}_{\mathrm{SBP}}$.

Three prominent peaks of power were frequently observed on this SBPV spectrum in different frequency bands, namely high-frequency (HF 1.3-2.5 Hz), mid-frequency (MF 0.33-0.73 Hz) and low-frequency components (LF $0.04-0.31 \mathrm{~Hz}$ ). In this study, averaged power values within each component over successive 5-min periods were employed as instantaneous power values for each frequency component at a given time point. On comparing SBPV and the variability of diastolic BP, we found that the power of LF and MF was similar in both variabilities, but that of HF was more prominent in SBPV in our pilot study; this result is consistent with that reported by other studies $[21,22]$. Therefore, we used the variability of $\mathrm{TS}_{\mathrm{SBP}}$ in this study.

Experimental protocol

\section{Experiment 1: Effects of isoflurane anesthesia on cardiovascular parameters}

Data were recorded in 5 rats in the anesthetized and unanesthetized states. In the experiment conducted in the unanesthetized state, a rat was kept singly in an acrylic cage (W 300 mm, H 130 mm, D 205 mm). After recording baseline data for $10 \mathrm{~min}$ during the stabilization period, we administered atropine $(10 \mathrm{mg} / \mathrm{kg}, n=5)$ or phentolamine $(10 \mathrm{mg} / \mathrm{kg}, n=5)$ intraperitoneally. We employed 10-min data for 10-20 min after each injection.

Experiments in the anesthetized state were performed as follows: (1) anesthesia was induced in the rats with $1.3 \%$ isoflurane in oxygen and an oral tube was attached, (2) the concentration of anesthesia was changed and maintained at $0.5-0.9 \%$ isoflurane in oxygen, (3) after recording 10-min data as baseline (approximately $20 \mathrm{~min}$ after administration of $0.5-0.9 \%$ isoflurane anesthesia), we administered atropine $(10 \mathrm{mg} / \mathrm{kg}, n=5)$ or phentolamine $(10 \mathrm{mg} / \mathrm{kg}$, $n=5$ ). The value after the administration was averaged 10-min data for 10-20 min after each administration.

SBPV was analyzed using the 10-min baseline data recorded before administration in the anesthetized $(n=5)$ and unanesthetized state $(n=5)$. SBP, HR and each component of SBPV were computed from the BP wave, as described above.

\section{Experiment 2: Effects of autonomic blockers on cardiovascular parameters in conscious rats at rest}

A rat was kept singly in the same acrylic cage as that used in Experiment 1. After recording baseline data for $20 \mathrm{~min}$ during the stabilization period, we administered phentolamine $(10 \mathrm{mg} / \mathrm{kg}, n=10)$ or atenolol $(10 \mathrm{mg} / \mathrm{kg}, n=10)$ 
intraperitoneally as a first injection. A second injection was done 35-40 min after the first injection: atenolol $(10 \mathrm{mg} /$ $\mathrm{kg}, n=5)$ or atropine $(10 \mathrm{mg} / \mathrm{kg}, n=5)$ after phentolamine treatment; phentolamine $(10 \mathrm{mg} / \mathrm{kg}, n=5)$ or atropine $(10 \mathrm{mg} / \mathrm{kg}, n=5)$ after atenolol treatment. We then calculated and averaged the 20-min data determined during the 10-30 min period after each injection.

\section{Experiment 3: Effects of autonomic blockers} on cardiovascular parameters in conscious rats during exposure to low ambient temperature (LT-exposure)

A rat was kept singly in the same acrylic cage as that used in Experiment 1 placed inside a box made of polystyrene foam (W $350 \mathrm{~mm}, \mathrm{H} 210 \mathrm{~mm}$, D $280 \mathrm{~mm}$ ). After recording baseline data for $30 \mathrm{~min}$, crushed ice was placed in the space between the cage and the box. The ambient temperature in the cage fell to approximately $9^{\circ} \mathrm{C}$ from $23^{\circ} \mathrm{C}$ in about $20 \mathrm{~min}$ and was maintained at approximately $9^{\circ} \mathrm{C}$ for 70 min (total LT-exposure, $90 \mathrm{~min}$ ). After LT-exposure, the ice was removed, and the temperature returned to the prior level in approximately $25-30 \mathrm{~min}$. In the control group, LT-exposure was performed as described above in rats that were not administered an autonomic blocker $(n=9)$. In the autonomic blocker groups, the rats were administered phentolamine (10 mg/kg, i.p., $n=5)$, lowdose atenolol $(1 \mathrm{mg} / \mathrm{kg}$, i.p., $n=5)$ or high-dose atenolol $(10 \mathrm{mg} / \mathrm{kg}$, i.p., $n=5) 30 \mathrm{~min}$ before LT-exposure. In a pilot study, we confirmed that, when the ambient temperature was changed $\left(20,15,9,7^{\circ} \mathrm{C}\right)$, HR showed a temperature-dependent increase and that there was no significant change in HR-increase at 9 and $7^{\circ} \mathrm{C}$. Therefore, we presume that the LT-exposure we used $\left(9^{\circ} \mathrm{C}\right)$ may be efficient enough to induce high sympathetic activation.

Baseline cardiovascular parameters measured over $30 \mathrm{~min}$ before LT-exposure were averaged. We calculated 90-min data recorded throughout LT-exposure because the gradual decrease in temperature induced an ANS response.

\section{Statistical analysis}

All data in Experiment 1 are presented as mean \pm SEM. SBP and HR in Experiments 2 and 3 are presented as box (median \pm interquartile range) and whisker (5 and 95 percentile values) plots, and data values for each SBPV component are expressed as mean \pm SEM. A paired $t$ test was employed to compare the data before and after drug administration, data recorded in the anesthetized and unanesthetized states in Experiment 1, before and after administration of the first drug in Experiment 2, and before and during LT-exposure (control) in Experiment 3. Twoway analysis of variance (ANOVA) and Holm's post hoc tests were utilized to compare treatments with drugs in Experiment 2. To compare all LT-exposure groups in Experiment 3, one-way ANOVA and Holm's post hoc tests were utilized. Differences were considered statistically significant at the $p<0.05$ level.

\section{Results}

This experiment revealed pharmacologically that MF and HF components of systolic arterial blood pressure variability (SBPV) changed along with $\alpha$ - and $\beta$-adrenoceptormediated sympathetic functions, respectively, in conscious rats. Before describing these results, we first report on the effects of isoflurane anesthesia on ANS.

Effects of isoflurane anesthesia on autonomic nervous function

In the anesthetized state, HR and SBP before administration of autonomic blockers were significantly different from those in the unanesthetized state; HR was markedly higher and SBP was significantly lower under anesthesia (Fig. 1a, b; see baseline). Spectral analysis of SBP baseline data showed that LF and MF components of SBPV under anesthesia were significantly lower than those under unanesthesia (LF $p=0.0186$, MF $p=0.0191$ ) (Fig. 1c). $\mathrm{HF}$ did not differ in the anesthetized and unanesthetized states (Fig. 1c).

Changes in HR as a direct effect of atropine $(10 \mathrm{mg} / \mathrm{kg}$, i.p.), which is a parasympathetic blocker to the heart, are shown in Fig. 1a. In the unanesthetized state, atropine administration increased HR (293.7-397.2 bpm, $p=0.0002)$, as expected. In contrast, in the anesthetized state, HR remained unchanged from the baseline level after atropine administration; thus, it was significantly lower than that in the unanesthetized state. Under anesthesia, HR did not show the direct effect of atropine. Atropine also increased SBP $(110.4-123.2 \mathrm{mmHg}, p=0.0159)$ under unanesthesia, but had only an insignificant effect on SBP under anesthesia (data not shown).

In the unanesthetized state, treatment with phentolamine (10 mg/kg, i.p.) as an $\alpha$-adrenoceptor-mediated sympathetic blocker significantly decreased SBP $(113.9-81.3 \mathrm{mmHg}$, $p=0.0099)$ and increased HR (286.3-399.4 bpm, $p=0.0009$ ), which is similar to the response of HR to atropine (Fig. 1a, b). In contrast, in the anesthetized state, phentolamine administration did not induce an increase in HR, despite a significant decrease in SBP (90.0 $72.7 \mathrm{mmHg}, p=0.0059$ ) (Fig. 1b). Anesthesia induced changes of baseline in SBP and HR, and also strongly affected HR when the $\alpha$-adrenoceptor blocker was treated. 

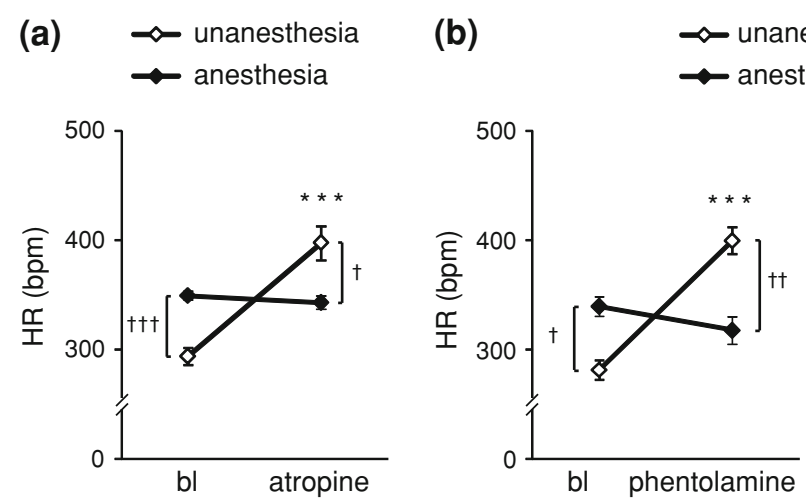

Fig. 1 Different effects of the anesthetized (using isoflurane) and unanesthetized states on autonomic nervous function. a Effect of atropine $(10 \mathrm{mg} / \mathrm{kg}$, i.p.) on heart rate (HR). b Effects of phentolamine $(10 \mathrm{mg} / \mathrm{kg}$, i.p.) on HR and systolic arterial blood pressure (SBP). c Differences in SBP variability (SBPV; 10-min recording data) at baseline (bl). Anesthetized state, $n=5$; unanesthetized state, $n=5$. Data are presented as mean \pm SEM. ${ }^{*} p<0.05, * * p<0.01$

Effects of autonomic blockers on cardiovascular parameters in conscious rats at rest

Figure 2 displays typical changes in cardiovascular parameters with subsequent administration of atenolol $(10 \mathrm{mg} / \mathrm{kg}$, i.p.) after phentolamine treatment $(10 \mathrm{mg} / \mathrm{kg}$, i.p. $)$ in a conscious rat (Experiment 2). Phentolamine treatment decreased SBP and increased HR, as described in the results obtained for the unanesthetized state in Fig. 1b. In terms of frequency components, MF decreased and LF slightly declined, whereas HF was considerably increased by phentolamine administration. These changes were kept stable from $10 \mathrm{~min}$ after the administration. Atenolol administration after phentolamine treatment induced sharp and significant decreases in the augmented responses of HR and HF, although other parameters were little affected (Fig. 2).

Averaged data from Experiment 2 are shown in Figs. 3 and 4. Three blockers were administered i.p. in sequential combinations: phentolamine $(10 \mathrm{mg} / \mathrm{kg})$ as an $\alpha$-adrenoceptor antagonist of vascular sympathetic control, atenolol $(10 \mathrm{mg} / \mathrm{kg})$ as a $\beta 1$-adrenoceptor antagonist of cardiac sympathetic control and atropine $(10 \mathrm{mg} / \mathrm{kg})$ as a muscarinic antagonist of cardiac parasympathetic control.

Figure 3 shows averaged data with responses of cardiovascular parameters following phentolamine pretreatment in conscious rats. Administration of phentolamine alone significantly decreased SBP $(108.1-79.6 \mathrm{mmHg}$, $p=0.0001)$ and increased HR $(282.8-429.6 \mathrm{bpm}$, $p<0.0001$ ) (Fig. 3); these results are consistent with those observed under unanesthesia in Experiment 1 (Fig. 1b). In terms of frequency components, phentolamine significantly reduced $\operatorname{LF}(p=0.0179)$ and $\mathrm{MF}(p=0.0023)$, and (c)

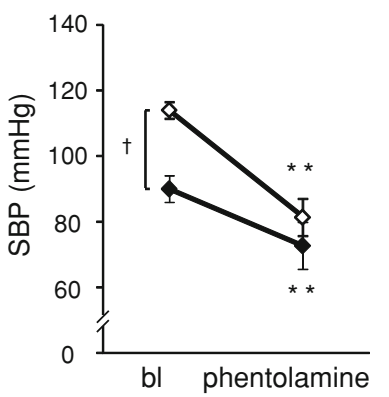

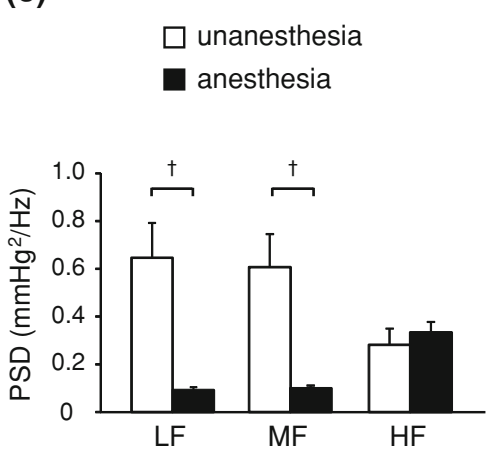

and $* * * p<0.001$ when compared with bl (paired $t$ test); $\dagger$ $p<0.05$, $\dagger \dagger p<0.01$ and $\dagger \dagger \dagger p<0.001$ compared with the unanesthetized and anesthetized states (paired $t$ test). PSD power spectrum density, $L F$ low frequency component, $M F$ middle frequency component, $H F$ high frequency component. All abbreviations in subsequent figures are the same as in this figure
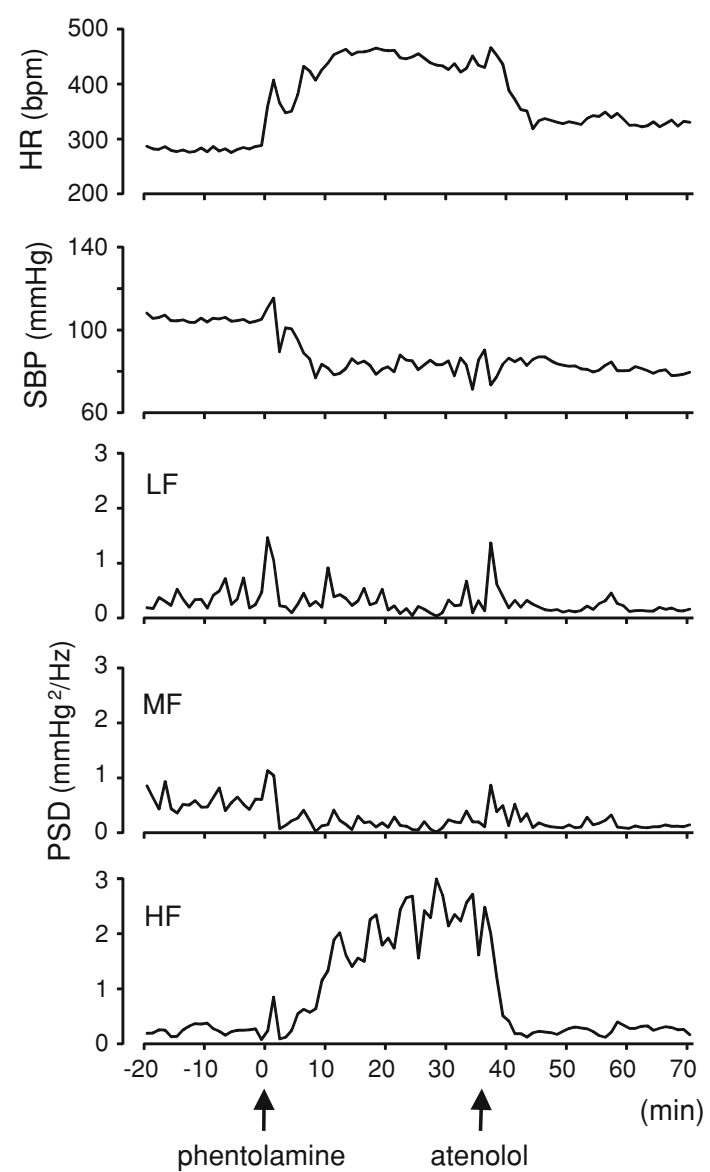

Fig. 2 Representative data of the time course of changes in cardiovascular parameters following atenolol administration after phentolamine treatment in a conscious rat. Arrows indicate the timing of injections of phentolamine (10 mg/kg, i.p.) and atenolol $(10 \mathrm{mg} /$ $\mathrm{kg}$, i.p.) 

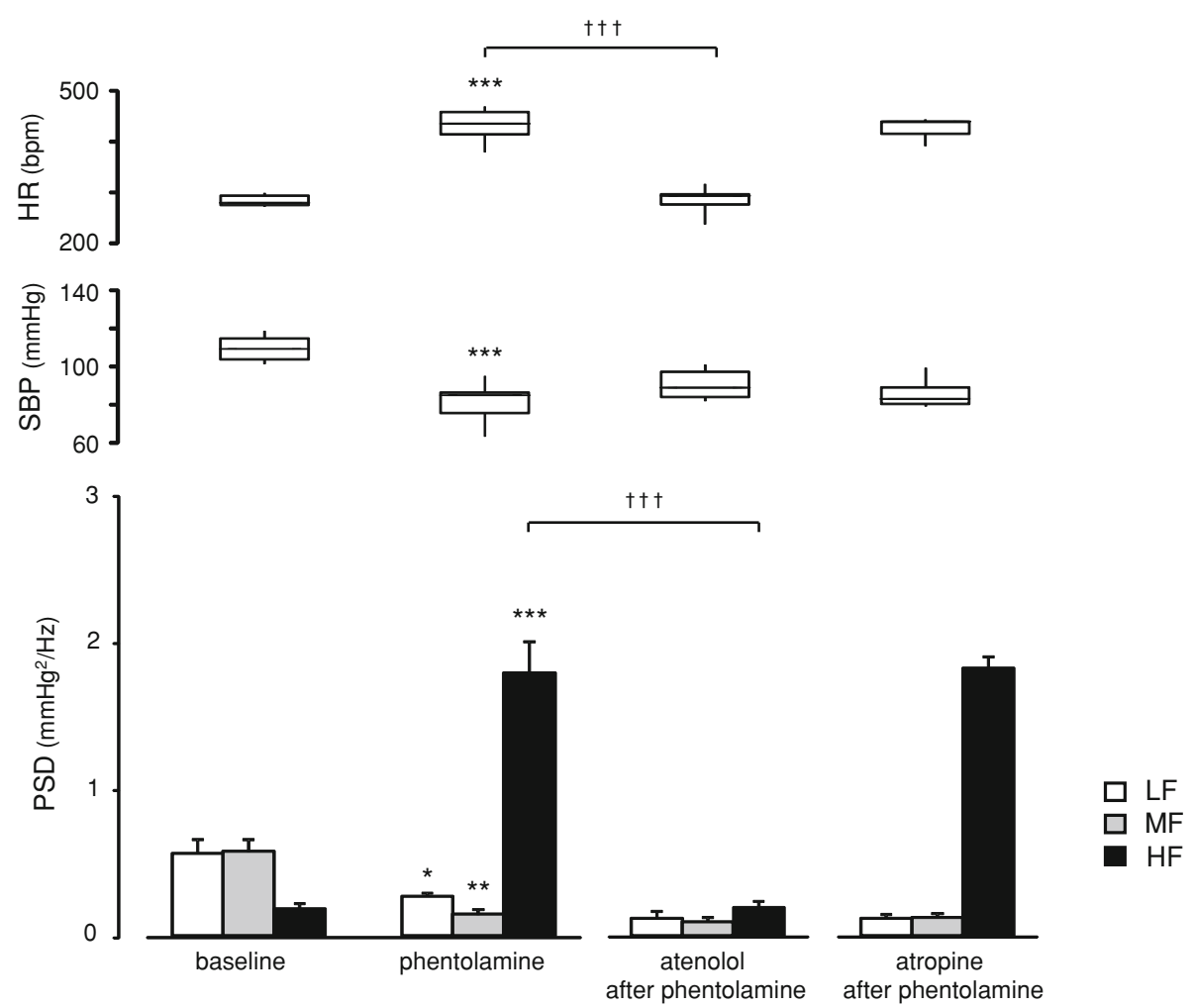

Fig. 3 Effects of subsequent administration of atenolol or atropine after phentolamine treatment on cardiovascular parameters in conscious rats: baseline: before treatments $(n=10)$; phentolamine: after treatment with phentolamine $(10 \mathrm{mg} / \mathrm{kg}$, i.p., $n=10)$ alone; atenolol after phentolamine: after atenolol administration $(10 \mathrm{mg} / \mathrm{kg}$, i.p., $n=5$ ) following phentolamine treatment; atropine after phentolamine: after atropine administration $(10 \mathrm{mg} / \mathrm{kg}$, i.p., $n=5)$ following phentolamine treatment. HR and SBP data are presented as box (median \pm interquartile range) and whisker (5 and 95 percentile

inversely augmented HF $(p<0.0001)$ (Fig. 3). The increases in HR and HF were markedly suppressed by subsequent treatment with atenolol (HR 454.7-288.8 bpm, $p<0.0001$, HF $\left.2.508-0.193 \mathrm{mmHg}^{2} / \mathrm{Hz}, \quad p<0.0001\right)$ (Figs. 2, 3), but not with atropine (Fig. 3). Atropine administration after phentolamine treatment had only an insignificant effect on all parameters.

Effects of preceding treatment with atenolol on cardiovascular parameters in conscious rats are indicated in Fig. 4. Treatment with atenolol alone led to a decrease in HR (288.5-264.8 bpm, $p=0.0181$ ), but did not affect SBP. In terms of frequency components, atenolol induced a small but significant decrease in HF $(p=0.0175)$, though no change was observed in LF and MF (Fig. 4). Phentolamine administration after atenolol treatment decreased SBP (111.4-83.3 mmHg, $p=0.0008)$ in a manner similar to that observed when phentolamine was administered alone (Figs. 1b, 2, 3); however, it did not change HR. As for the frequency components, phentolamine administration after atenolol treatment decreased MF $(p=0.026)$ in the same way as that observed when phentolamine was

values) plots, and SBPV data are presented as mean \pm SEM. Averaged 20-min data were used as baseline data; data of treatment with autonomic blockers were averaged from 20-min data recorded during the 10-30 min after each injection. * $p<0.05, * * p<0.01$ and $*_{* *}^{*} p<0.001$ compared with baseline (paired $t$ test); $\dagger p<0.05$, $\dagger \dagger$ $p<0.01$ and $\dagger \dagger \uparrow p<0.001$ when compared with first and second treatment [two-way analysis of variance (ANOVA) and Holm's post hoc test]

administered alone (Figs. 1b, 2, 3), but, unlike phentolamine alone, it did not increase HF (Fig. 4). Augmentation of HF after phentolamine administration occurred only when phentolamine was administered alone, but not when the rats were pretreated with atenolol (Figs. 3, 4), i.e., HF was enhanced only when $\beta$-adrenoceptor was intact. In addition, MF decreased only when phentolamine was injected, regardless of pretreatment with other blockers (Figs. 3, 4). Atropine administration after atenolol treatment increased HR (260.8-329.5 bpm, $p=0.0029)$, but the other parameters remained unchanged (Fig. 4). Throughout this experiment, atropine had little effect on each SBPV component, even after preceding treatment with phentolamine or atenolol (Figs. 3, 4).

Effects of autonomic blockers on cardiovascular parameters during exposure to low ambient temperature (LT-exposure)

The results from Experiment 2 indicated that the changes in MF and HF may be interrelated with administration of 


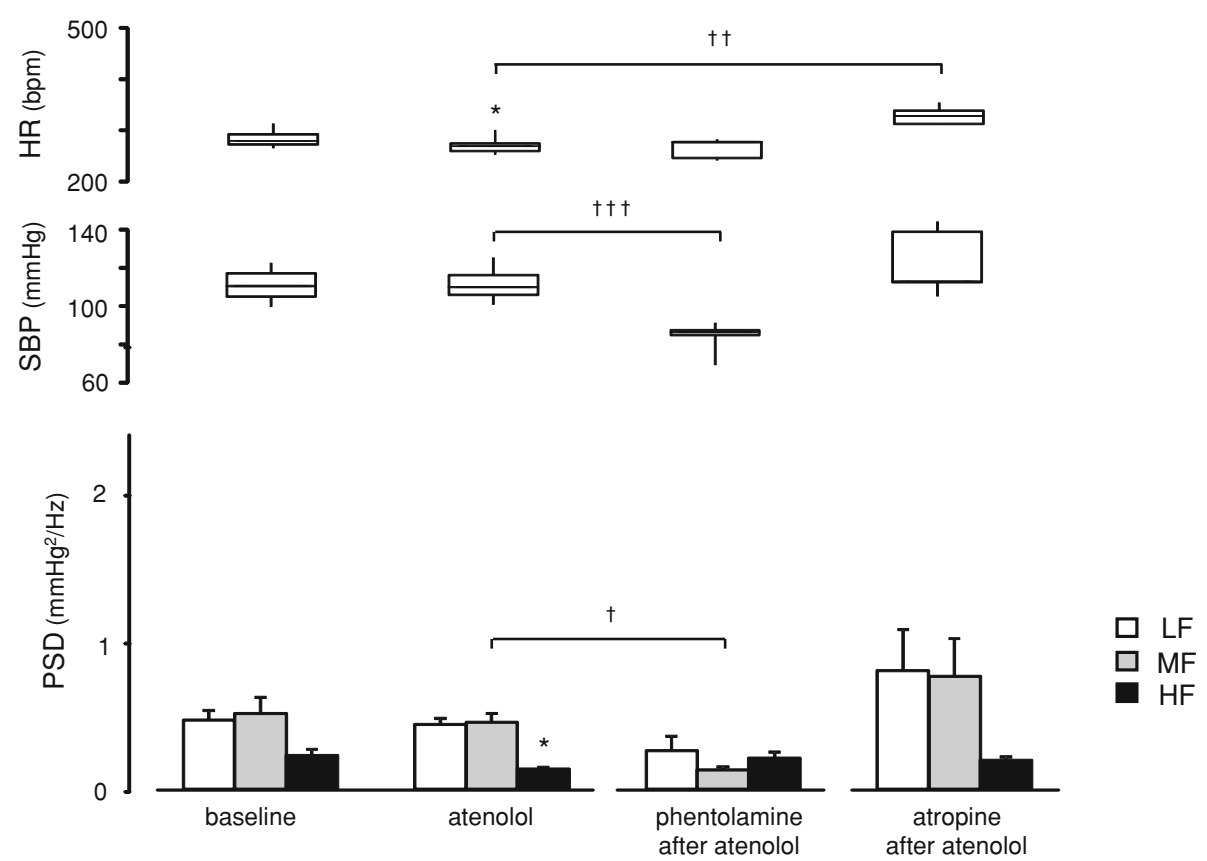

Fig. 4 Effects of subsequent administration of phentolamine or atropine after atenolol treatment on cardiovascular parameters in conscious rats: baseline: before treatments $(n=10)$; atenolol: after treatment with atenolol $(10 \mathrm{mg} / \mathrm{kg}$, i.p., $n=10)$ alone; phentolamine after atenolol: after phentolamine administration $(10 \mathrm{mg} / \mathrm{kg}$, i.p., $n=5$ ) following atenolol treatment; atropine after atenolol: after atropine administration $(10 \mathrm{mg} / \mathrm{kg}$, i.p., $n=5)$ following atenolol treatment. HR, SBP, and SBPV data are presented as in Fig. 3. In this figure, 20-min data were employed in the same manner as those used in Fig. 3. $* p<0.05, * * p<0.01$ and $* * * p<0.001$ compared with baseline (paired $t$ test); $\dagger p<0.05$, $\dagger \dagger p<0.01$ and $\dagger \dagger \dagger p<0.001$ when compared with first and second treatment [two-way analysis of variance (ANOVA) and Holm's post hoc test]

in 1 vs. $10 \mathrm{mg} / \mathrm{kg}]$. Other parameters, SBP, LF and MF in the atenolol pretreatment group were not significantly different from those in the control group (Fig. 5).

In summary, MF was attenuated after phentolamine administration ( $\alpha$-adrenoceptor blocker), and HF was reduced after atenolol administration ( $\beta$-adrenoceptor blocker).

\section{Discussion}

In this study, we investigated HR and SBP responses to three kinds of autonomic blockers under resting and systemic sympathetic activated conditions. Involvement of sympathetic cardiovascular function in SBPV frequency components were explored pharmacologically through the effects of these blockers. We found that each SBPV component has a specific correspondence with vasomotor or cardiac sympathetic function, respectively, in conscious rats.

Suppression of the autonomic nervous system by isoflurane anesthesia

Many studies regarding the involvement of ANS in SBPV have been conducted in the anesthetized state [14-16] because 


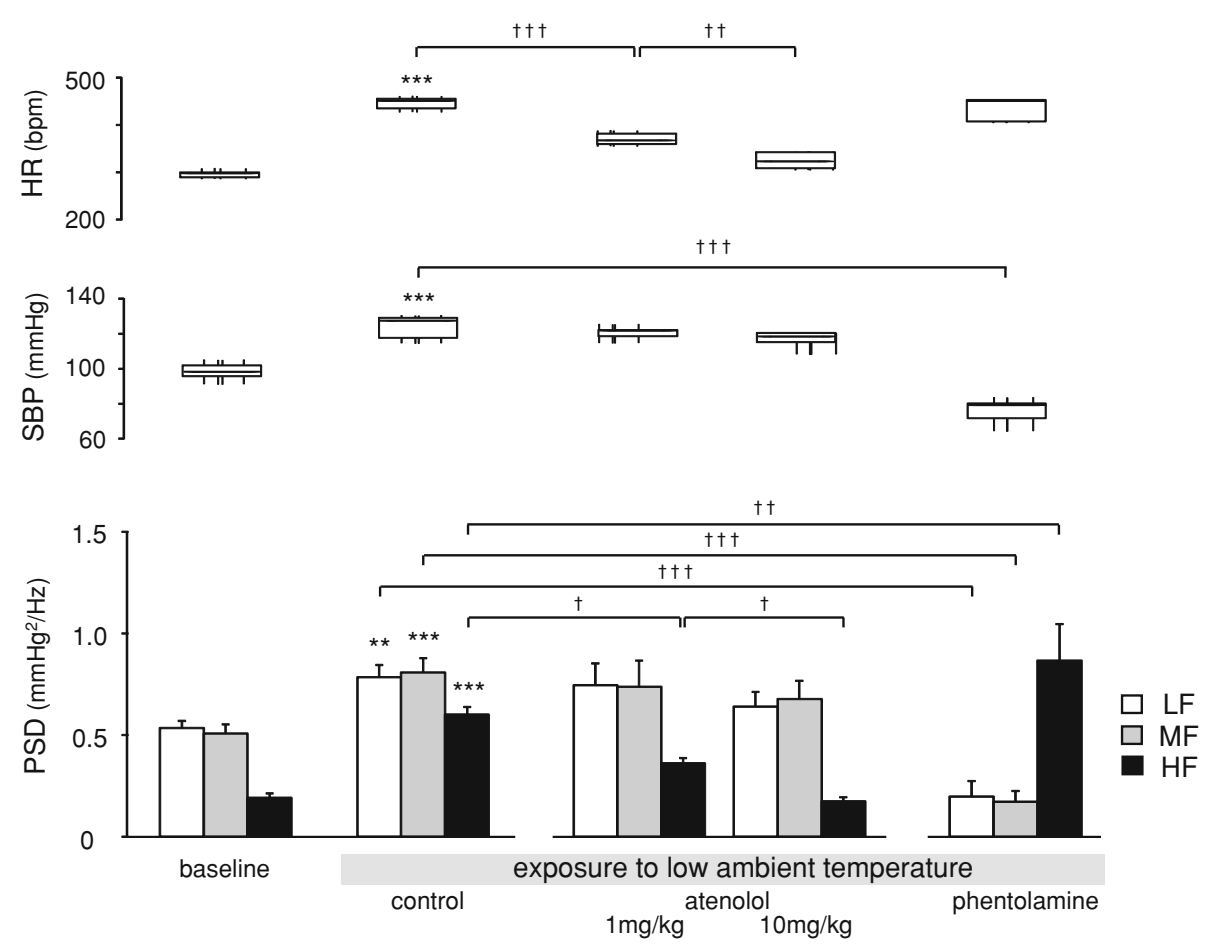

Fig. 5 Effects of autonomic blockers on cardiovascular parameters during exposure to low ambient temperature (LT-exposure): baseline: before LT-exposure $(n=9)$; control: during LT-exposure without an autonomic blocker $(n=9)$; atenolol: during LT-exposure after intraperitoneal (i.p.) injection of atenolol $(1 \mathrm{mg} / \mathrm{kg}, n=5$ or $10 \mathrm{mg} / \mathrm{kg}, n=5$ ); phentolamine: during LT-exposure after i.p. injection of phentolamine $(10 \mathrm{mg} / \mathrm{kg}, n=5)$. HR, SBP and SBPV

of the simple methods, but it may be important to examine natural responses in the conscious state, in particular, considering sequential measurements of changes in complicated pathological conditions such as chronic pain [18-20].

Isoflurane anesthesia is widely clinically used as an inhalant anesthetic because it is well tolerated. In this study, isoflurane anesthesia induced significant changes of baseline in HR and SBP; HR became higher and SBP became lower than under unanesthesia (Fig. 1a, b). In terms of frequency components of baseline data, the power of LF and MF components of SBPV in the anesthetized state significantly decreased compared to those in the unanesthetized state (Fig. 1c), thus supporting results of other studies [23, 24]. Lower frequency components such as LF and MF are generally considered to be attributed to the influence of sympathetic nervous function [22, 25]; therefore, our results suggest that isoflurane anesthesia may depress ANS function.

Pharmacological experiments conducted under isoflurane anesthesia indicated that atropine acting as a muscarinic receptor antagonist had no effect on HR (Fig. 1a). Given the result that HR in baseline under anesthesia was higher (Fig. 1a, b), isoflurane anesthesia may induce vagal suppression, confirming the results of other studies that data are presented as in Fig. 3. Averaged 30-min data were used as baseline data, and 90-min data throughout the exposure period were used for the LT-exposure groups. $* p<0.05,{ }^{* *} p<0.01$ and $* * * p<0.001$ compared with baseline and control (paired $t$ test); $\dagger$ $p<0.05$, $\dagger \dagger p<0.01$ and $\dagger \dagger \dagger p<0.001$ compared among LT-exposure groups [one-way analysis of variance (ANOVA) and Holm's post hoc test]

reported that respiratory sinus arrhythmia [26] and HR $[26,27]$ remained unchanged after surgical vagotomy in isoflurane-anesthetized rats. In addition, phentolamine administration ( $\alpha$-adrenoceptor antagonist) in this study induced a decrease in SBP regardless of the induction of anesthesia, but had no effect on HR in the anesthetized state (Fig. 1b). These results suggest that the central nervous system involved in baroreflex may also be depressed by isoflurane anesthesia.

Relationship between MF component and $\alpha$ adrenoceptor-mediated vasomotor function

At rest, MF in this study was reduced when phentolamine was administered as an $\alpha$-adrenoceptor blocker (Figs. 2, 3, 4,5 ), which is consistent with the results of other studies [9-12]. Phentolamine-induced decreases in MF occurred reliably, regardless of whether phentolamine was administered alone or in combination; MF was consistently unaffected by atenolol administration ( $\beta$-adrenoceptor antagonist). The MF component of SBPV in this study may have changed solely depending on vasomotor function via the $\alpha$-adrenoceptor, but not on cardiac sympathetic function. In our preliminary experiment, we confirmed that 
saline administration induced no significant changes in BP, HR and each component of SBPV (data not shown). Therefore, we did not use saline administration in a control group in this study.

Exposure to low ambient temperature (LT-exposure) in our experiment induced significant augmentation of SBP and HR, which is indicative of systemic sympathetic activation and also confirms the results of other studies [28]. MF of SBPV significantly increased during LT-exposure; however, phentolamine pretreatment suppressed augmentation of SBP and MF (Fig. 5). These results in a systemic sympathetic activated state also indicated a strong relationship between MF and vasomotor function.

The MF component of blood pressure variability (BPV) has been considered to be involved in vasomotor nerve activity via an arterial baroreflex in previous studies of humans [25] and animals [8, 10, 29]. However, the detailed characteristics of MF have remained unclear because the method of sequential administration of different types of autonomic antagonists at rest and in a sympathetic activated state has never been used. Our study revealed the relationship between $\mathrm{MF}$ and $\alpha$-adrenoceptor-mediated vasomotor function using this more comprehensive method.

Relationship between HF component and $\beta$-adrenoceptor-mediated cardiac sympathetic function

In this study, the $\alpha$-adrenoceptor blocker, which induced a decrease in SBP, profoundly enhanced the HF component of SBPV (Figs. 2, 3). A similar effect was observed by other authors [9, 11, 12] and has been considered to be attributed to the mechanical effect of respiration on intrathoracic pressure as blood vessels relax or dilate. Our results, however, showed that the enhanced-HF caused by the $\alpha$-adrenoceptor blocker was markedly suppressed by subsequent treatment with the $\beta$-adrenoceptor blocker (Fig. 3). In addition, results with the $\beta$-adrenoceptor blocker indicated that HF did not increase, even when SBP was decreased by the $\alpha$-adrenoceptor blocker (Fig. 4), i.e., once the $\beta$-adrenoceptor was blocked, either before or after the $\alpha$-adrenoceptor blocker was used, HF remained at a low level. These findings indicate that the HF component may be linked to $\beta$-adrenoceptor-mediated cardiac sympathetic function rather than the mechanical effects of respiration.

HF of SBPV is in the same frequency as that of heart rate variability (HRV), which is considered to correspond to respiratory fluctuations. The mechanism underlying the $\mathrm{HF}$ respiratory component in the $\mathrm{BP}$ wave causes controversy as to whether mechanical effects of respiration $[9,12,13]$ or involvement of ANS, particularly in the cardiac sympathetic nervous system [14-16], is involved; however, previous studies that indicated an association between cardiac sympathetic function and HF were performed in the anesthetized state. In our study using conscious animals, we found that HF component appears to be involved in $\beta$-adrenoceptormediated cardiac sympathetic function rather than respiration.

An increase in the HF component of SBPV in mechanically ventilated, anesthetized rats has been reported to be induced blood-loss-dependently by hemorrhage $[11,16]$, and the HF component is considered to be an index of blood volume status. Meanwhile, during gradation of hemorrhage or alleviation of myocardial preload, compensation via the sympathetic nervous system generally works as a circulation system. In the light of our results, augmentation of HF after $\alpha$-adrenoceptor blockade may reflect baroreceptor responses as compensatory activation of the $\beta$-adrenoceptor-mediated sympathetic nervous system caused by alleviation of the myocardial preload in the same manner as that observed during hemorrhage.

Atenolol administration alone in this study induced a small but significant decrease in HF (Fig. 4), and atropine administration after atenolol treatment had no effect on HF (Fig. 4). In addition, the amplifying effect of phentolamine on HF was reduced by subsequent treatment with atenolol but not with atropine (Fig. 3). It has been reported that respiratory-related arterial BPV in anesthetized rats is attenuated by the $\beta$-adrenoceptor blocker [14-16, 21], but not by vagotomy [14] or atropine [16]. Our results imply that there may be a negative involvement of the vagal function in the genesis of the HF component of SBPV.

We found that the augmented HF induced by LTexposure was dose-dependently suppressed by $\beta$-adrenergic blocker before exposure (Fig. 5). The HF component was enhanced under alleviation of myocardial preload by the $\alpha$-adrenoceptor blockade and also under augmentation of the preload by LT-exposure (systemic sympathetic activation), i.e., when the cardiac sympathetic nervous system was activated. The $\beta$-adrenergic system was indicated to be involved in the genesis of HF in this study. It may be possible that HF of SBPV is applied not only as a specific reflection of a baroreflex compensatory response, but also as a general marker of $\beta$-adrenergic sympathetic excitation. In this study, we activated the cardiac sympathetic nervous system indirectly (with use of phentolamine or LT-exposure). In order to establish the method to evaluate various pathological states, it may be necessary to perform further experiments under several conditions, such as using an autonomic agonist or specific animal model in which the $\beta$-adrenergic system is enhanced.

\section{Conclusion}

In this study, we showed that the MF and HF components of SBPV in conscious rats may reflect the activity of 
vasomotor and cardiac sympathetic nervous systems, respectively, during systemic sympathetic activation as well as during sympathetic deactivation by autonomic antagonists. Our results also demonstrated that isoflurane anesthesia depressed ANS. We suggest that the MF and HF components of SBPV may act as alternative means of monitoring vasomotor and cardiac sympathetic regulation in a conscious state. Further research is required concerning the link between nerve activity and each component in the unanesthetized states or changes in autonomic nervous function under complicated pathological conditions. Hopefully, such approaches will help enhance the functionality of current BP monitoring and improve our understanding of autonomic dysfunction.

Acknowledgments This study was supported by the Research Budget (2002-2008) of the Department of Algesiology, Aichi Medical University, donated by Pfizer Inc., a Grant-in-Aid for Scientific Research (C) (18613020, 20602009) from the Japan Society for the Promotion of Science, and academic research grants from the Daiko Foundation (2007) and from the Foundation for Oriental Medicine Research (2007). There were no conflicts of interest in the conducting of these experiments and reporting of results.

Open Access This article is distributed under the terms of the Creative Commons Attribution Noncommercial License which permits any noncommercial use, distribution, and reproduction in any medium, provided the original author(s) and source are credited.

\section{References}

1. Task Force of the European Society of Cardiology, the North American Society of Pacing, Electrophysiology (1996) Heart rate variability: standards of measurement, physiological interpretation and clinical use. Circulation 93:1043-1065

2. Pagani M, Lombardi F, Guzzetti S, Rimoldi O, Furlan R, Pizzinelli P, Sandrone G, Malfatto G, Dell'Orto S, Piccaluga E et al (1986) Power spectral analysis of heart rate and arterial pressure variabilities as a marker of sympatho-vagal interaction in man and conscious dog. Circ Res 59:178-193

3. Ori Z, Monir G, Weiss J, Sayhouni X, Singer DH (1992) Heart rate variability. Frequency domain analysis. Cardiol Clin 10:499-537

4. Kollai M, Koizumi K (1979) Reciprocal and non-reciprocal action of the vagal and sympathetic nerves innervating the heart. J Auton Nerv Syst 1:33-52

5. Ponchon P, Elghozi JL (1997) Contribution of humoral systems to the short-term variability of blood pressure after severe hemorrhage. Am J Physiol 273:R58-R69

6. Brown DR, Brown LV, Patwardhan A, Randall DC (1994) Sympathetic activity and blood pressure are tightly coupled at $0.4 \mathrm{~Hz}$ in conscious rats. Am J Physiol 267:R1378-R1384

7. Cheng Y, Cohen B, Orea V, Barres C, Julien C (2004) Baroreflex control of renal sympathetic nerve activity and spontaneous rhythms at Mayer wave's frequency in rats. Auton Neurosci 111:80-88

8. Julien C, Chapuis B, Cheng Y, Barres C (2003) Dynamic interactions between arterial pressure and sympathetic nerve activity: role of arterial baroreceptors. Am J Physiol Regul Integr Comp Physiol 285:R834-R841
9. Japundzic N, Grichois ML, Zitoun P, Laude D, Elghozi JL (1990) Spectral analysis of blood pressure and heart rate in conscious rats: effects of autonomic blockers. J Auton Nerv Syst 30:91-100

10. Rubini R, Porta A, Baselli G, Cerutti S, Paro M (1993) Power spectrum analysis of cardiovascular variability monitored by telemetry in conscious unrestrained rats. J Auton Nerv Syst 45:181-190

11. Baujard C, Ponchon P, Elghozi JL (1996) Effects of graded hemorrhage on short-term variability of blood pressure in conscious rats. Fundam Clin Pharmacol 10:511-517

12. Gonzalez Gonzalez J, Cordero Valeriano JJ, Feria Rodriguez M (1995) Autonomic mediation of short-term cardiovascular oscillations after acute hemorrhage in conscious rats. J Auton Nerv Syst 55:123-130

13. Zhang R, Iwasaki K, Zuckerman JH, Behbehani K, Crandall CG, Levine BD (2002) Mechanism of blood pressure and R-R variability: insights from ganglion blockade in humans. J Physiol 543:337-348

14. Kuo TB, Yang CC, Chan SH (1996) Transfer function analysis of ventilatory influence on systemic arterial pressure in the rat. Am J Physiol 271:H2108-H2115

15. Yang CC, Kuo TB (1999) Assessment of cardiac sympathetic regulation by respiratory-related arterial pressure variability in the rat. J Physiol 515(Pt 3):887-896

16. Lai HY, Yang CC, Huang FY, Lee Y, Kuo YL, Kuo TB (2003) Respiratory-related arterial pressure variability as an indicator of graded blood loss: involvement of the autonomic nervous system. Clin Sci (Lond) 105:491-497

17. Wasner G, Schattschneider J, Heckmann K, Maier C, Baron R (2001) Vascular abnormalities in reflex sympathetic dystrophy (CRPS I): mechanisms and diagnostic value. Brain 124:587-599

18. Jin Y, Sato J, Yamazaki M, Omura S, Funakubo M, Senoo S, Aoyama M, Mizumura K (2008) Changes in cardiovascular parameters and plasma norepinephrine level in rats after chronic constriction injury on the sciatic nerve. Pain 135:221-231

19. Yoshimoto T, Eguchi K, Sakurai H, Ohmichi Y, Hashimoto T, Morimoto A, Ohmichi M, Yamaguchi Y, Kumazawa T (2008) Sympathetic dysfunction in the cast-immobilization chronic pain model. Abstracts of 12th World Congress on pain, vol 28

20. Yamaguchi Y, Hashimoto T, Sakurai H, Yoshimoto T, Ohmichi Y, Morimoto A, Harimoto K, Ohmichi M, Eguchi K, Kumazawa T (2011) Low rather than high dose lipopolysaccharide 'priming' of muscle provides an animal model of persistent elevated mechanical sensitivity for the study of chronic pain. Eur J Pain (in press)

21. Yang CC, Kuo TB (2000) Impact of pulse pressure on the respiratory-related arterial pressure variability and its autonomic control in the rat. Pflugers Arch 439:772-780

22. Cerutti C, Gustin MP, Paultre CZ, Lo M, Julien C, Vincent M, Sassard J (1991) Autonomic nervous system and cardiovascular variability in rats: a spectral analysis approach. Am J Physiol 261:H1292-H1299

23. Kawase M, Komatsu T, Nishiwaki K, Kobayashi M, Kimura T, Shimada Y (2002) Heart rate variability and arterial blood pressure variability show different characteristic changes during hemorrhage in isoflurane-anesthetized, mechanically ventilated dogs. Anesth Analg 94:16-21

24. Wang H, Kuo TB, Chan SH, Tsai TH, Lee TY, Lui PW (1996) Spectral analysis of arterial pressure variability during induction of propofol anesthesia. Anesth Analg 82:914-919

25. Pagani M, Montano N, Porta A, Malliani A, Abboud FM, Birkett C, Somers VK (1997) Relationship between spectral components of cardiovascular variabilities and direct measures of muscle sympathetic nerve activity in humans. Circulation 95:1441-1448

26. Tzeng YC, Galletly DC, Larsen PD (2005) Paradoxical respiratory sinus arrhythmia in the anesthetized rat. Auton Neurosci $118: 25-31$ 
27. Marano G, Grigioni M, Tiburzi F, Vergari A, Zanghi F (1996) Effects of isoflurane on cardiovascular system and sympathovagal balance in New Zealand white rabbits. J Cardiovasc Pharmacol 28:513-518

28. Raven PB, Niki I, Dahms TE, Horvath SM (1970) Compensatory cardiovascular responses during an environmental cold stress, 5 degrees C. J Appl Physiol 29:417-421
29. Cerutti C, Barres C, Paultre C (1994) Baroreflex modulation of blood pressure and heart rate variabilities in rats: assessment by spectral analysis. Am J Physiol 266:H1993-H2000 